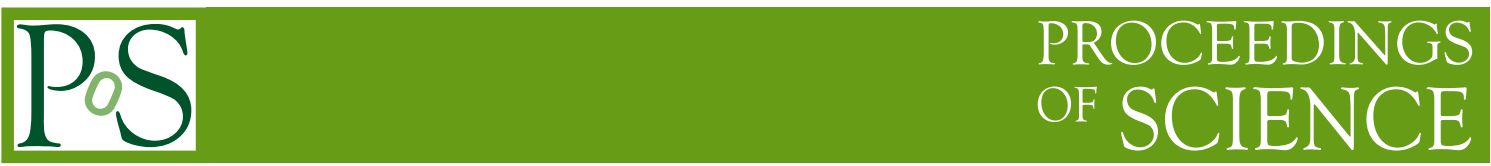

\title{
CP Violation Theory
}

\section{UTfit Collaboration}

\section{A.J. Bevan and M. Bona}

Queen Mary, University of London, Mile End Road, London E1 4NS, United Kingdom

\section{Ciuchini}

INFN, Sezione di Roma Tre, Via della Vasca Navale 84, I-00146 Roma, Italy

\section{Derkach and M. Pierini}

CERN, CH-1211 Geneva 23, Switzerland

\section{E. Franco and Luca Silvestrini*}

INFN, Sezione di Roma, Piazzale A. Moro 2, I-00185 Roma, Italy

E-mail: Luca.Silvestrini@romal.infn.it

\section{Lubicz and C. Tarantino}

Dipartimento di Fisica, Università di Roma Tre and INFN, Sez. di Roma Tre, Via della Vasca Navale 84, I-00146 Roma, Italy

\section{G. Martinelli}

INFN, Sezione di Roma, Piazzale A. Moro 2, I-00185 Roma, Italy and SISSA-ISAS, Via Bonomea 265, I-34136 Trieste, Italy

\section{F. Parodi and C. Schiavi}

Dipartimento di Fisica, Università di Genova and INFN, Via Dodecaneso 33, I-16146 Genova, Italy

\section{Sordini}

IPNL-IN2P3, 4 Rue Enrico Fermi, F-69622 Villeurbanne Cedex, France

\section{A. Stocchi}

Laboratoire de l'Accélérateur Linéaire, IN2P3-CNRS et Université de Paris-Sud, BP 34, F-91898 Orsay Cedex, France

\section{Vagnoni}

INFN, Sezione di Bologna, Via Irnerio 46, I-40126 Bologna, Italy

We discuss CP violation in meson-antimeson mixing in the SM and beyond and derive constraints on the scale of new physics.

14th International Conference on B-Physics at Hadron Machines,

April 8-12, 2013

Bologna, Italy

${ }^{*}$ Speaker. 


\section{Introduction}

The Standard Model (SM) of electroweak and strong interactions is extremely successful in describing all presently available experimental data. However, its validity can extend at most to energies of the order of the Planck scale, where gravity comes into play. Let us therefore consider the SM as an effective theory valid up to a scale $\Lambda$. We can then write the SM Lagrangian as

$$
\mathscr{L}=C_{2} \Lambda^{2} H^{\dagger} H+\lambda\left(H^{\dagger} H\right)^{2}+\mathscr{L}_{\text {gauge }}+\mathscr{L}_{\text {Yukawa }}+\sum_{d=5}^{\infty} \sum_{i=1}^{n_{d}} \frac{C_{d}^{i}}{\Lambda^{(d-4)}} O_{d}^{i},
$$

where $O_{d}^{i}$ is a generic gauge-invariant operator of dimension $d$. Now, it turns out that the Lagrangian truncated at $d \leq 4$ has some very important "accidental" symmetries that are violated by $O_{d>4}^{i}$. Most notable examples of such symmetries are given by baryon and lepton number conservation. The agreement of the SM with experimental data would suggest a very high value of $\Lambda$, so that the breaking of SM accidental symmetries gets strongly suppressed by the inverse powers of $\Lambda$ in front of the higher-dimensional operators. However, we see from the first term in eq. (1.1) that $C_{2} \Lambda$ controls the scale of electroweak symmetry breaking. Thus, unless we are willing to accept an extremely small value of $C_{2}$ (which means an extremely large amount of fine-tuning, since radiative corrections within the effective theory naturally generate $C_{2} \sim \mathscr{O}(1)$ ), we are forced to consider values of the New Physics (NP) scale $\Lambda$ not too far above the electroweak scale. But then the SM accidental symmetries require that NP has a peculiar structure, so that the cofficients of symmetrybreaking higher dimensional operators are strongly suppressed and the phenomenological success of the SM remains unscathed. Turning the argument around, the coefficients of those higher dimensional operators that break SM accidental symmetries provide the most stringent constraints on the NP scale and couplings (or better, on a combination thereof).

Let us now concentrate on two accidental symmetries of the SM: i) the absence of tree-level Flavour Changing Neutral Currents (FCNC), and the GIM suppression of loop-mediated FCNC; ii) The absence of tree-level CP violation in weak interactions. These accidental symmetries ensure that flavour physics is extremely sensitive to NP. In particular, CP violation in quark weak interactions in the SM is governed by one single phase in the Cabibbo-Kobayashi-Maskawa (CKM) mixing matrix, leading to stringent correlations between all $\mathrm{CP}$ violating observables. A very useful tool to study these correlations is given by the so-called Unitarity Triangle Analysis (UTA). The unitarity of the CKM matrix implies several triangular relations; in particular, one of these relations defines a triangle whose sides and angles are connected to several observables in flavour physics. Since all CP violating observables are connected to the phase of the CKM matrix, they all translate into a constraint on the apex of the UT. Furthermore, the UTA allows to combine in a coherent way all constraints coming from both $\mathrm{CP}$-conserving and $\mathrm{CP}$-violating processes. In the left plot in Figure 1 we present the result of the UTA in the SM. We obtain at $68 \%$ probability $\bar{\rho}=0.132 \pm 0.021$ and $\bar{\eta}=0.350 \pm 0.014$.

\section{CP violation in meson-antimeson mixing}

Neutral meson mixing is governed by the transition matrix element between $M^{0}$ and $\bar{M}^{0}$ mesons, which can be parameterized in terms of two fundamental matrix elements: $M_{12}$, which 


\begin{tabular}{|l||c|c|c|c|c|}
\hline Parameter & $C_{\varepsilon_{K}}$ & $C_{B_{d}}$ & $\phi_{B_{d}}\left[^{\circ}\right]$ & $C_{B_{s}}$ & $\phi_{B_{s}}\left[^{\circ}\right]$ \\
\hline Value & $1.08 \pm 0.18$ & $1.01 \pm 0.15$ & $-2.2 \pm 3.7$ & $1.03 \pm 0.10$ & $-0.84 \pm 2.47$ \\
\hline
\end{tabular}

Table 1: Numerical results (at $68 \%$ probability) for the NP parameters in $K$ and $B_{q}$ mixing.

for $B_{q}$ and for the CP-violating part of $K^{0}$ mixing is dominated by the exchange of virtual heavy states (top quarks and possibly new heavy particles), and $\Gamma_{12}$, which is dominated by the tree-level exchange of on-shell intermediate states. We assume here and in the following that NP is a negligible correction to tree-level processes. We can therefore write the relevant amplitudes in terms of SM ones as follows:

$$
\begin{aligned}
M_{12, M}^{\text {full }} & =\left\langle M\left|\mathscr{H}^{\text {eff }}\right| \bar{M}\right\rangle=M_{12, M}^{\mathrm{SM}}+M_{12, M}^{\mathrm{NP}}=C_{M} e^{i \phi_{M}} M_{12, M}^{\mathrm{SM}} \\
\Gamma_{12, M}^{\mathrm{full}} & =\Gamma_{12, M}^{\mathrm{SM}}+\text { penguin effects } .
\end{aligned}
$$

Notice that $\operatorname{Im}\left(\Gamma_{12, B_{q}}^{\mathrm{SM}} / M_{12, B_{q}}^{\mathrm{SM}}\right) \sim 0$ due to GIM suppression, since $M_{12, B_{q}}^{\mathrm{SM}} \propto\left(V_{t b} V_{t q}^{*}\right)^{2}$ and $\Gamma_{12, B_{q}}^{\mathrm{SM}} \propto$ $\left(V_{t b} V_{t q}^{*}\right)^{2}+$ GIM-suppressed terms. On the other hand, in the standard CKM parameterization,

$\operatorname{Arg}\left(M_{12, B_{d}}^{\mathrm{SM}}\right)=2 \beta \sim \mathscr{O}(1)$, but $\operatorname{Arg}\left(M_{12, B_{s}}^{\mathrm{SM}}\right)=-2 \beta_{s} \sim \mathscr{O}\left(10^{-2}\right)$ and $\operatorname{Arg}\left(M_{12, D^{0}}^{\mathrm{SM}}, \Gamma_{12, D^{0}}^{\mathrm{SM}}\right)<O\left(10^{-2}\right)$.

From experiments we can extract the following combinations of the above parameters in the $K$ and $B$ sectors:

$$
\begin{aligned}
& \Delta m_{B_{q}}=2\left|M_{12, q}^{\mathrm{full}}\right|=C_{B_{q}} \Delta m_{B_{q}}^{\mathrm{SM}}, \quad \frac{\Delta \Gamma_{q}}{\Delta m_{B_{q}}}=\operatorname{Re} \frac{\Gamma_{12, q}^{\text {full }}}{M_{12, q}^{\text {full }}} \sim \frac{\Delta \Gamma_{q}^{\mathrm{SM}}}{\Delta m_{B_{q}}^{\mathrm{SM}}} \frac{\cos 2 \phi_{B_{q}}}{C_{B_{q}}}, \\
& A_{\mathrm{SL}}^{q}=\operatorname{Im} \frac{\Gamma_{12, q}^{\mathrm{full}}}{M_{12, q}^{\text {full }}} \sim-\frac{\Delta \Gamma_{q}^{\mathrm{SM}}}{\Delta m_{B_{q}}^{\mathrm{SM}}} \frac{\sin 2 \phi_{B_{q}}}{C_{B_{q}}} \sim-\frac{\Delta \Gamma_{q}}{\Delta m_{B_{q}}} \tan 2 \phi_{B_{q}}, \\
& S_{J / \Psi K} \sim \sin 2\left(\beta+\phi_{B_{d}}\right), \quad S_{J / \Psi \phi} \sim \sin 2\left(-\beta_{S}+\phi_{B_{s}}\right), \quad \varepsilon_{K} \sim C_{\varepsilon_{K}} \varepsilon_{K}^{\mathrm{SM}} .
\end{aligned}
$$

To exploit the full constraining power of the measurements in eq. (2.2) we must obtain a NP-free determination of CKM parameters, so that we can compute the SM mixing amplitudes. To this aim, we use tree-level processes: semileptonic $B$ decays determine $\left|V_{u b}\right|$ and $\left|V_{c b}\right|, B \rightarrow D K$ decays determine the angle $\gamma$ of the Unitarity Triangle (UT) and $B \rightarrow \pi \pi, \pi \rho$ and $\rho \rho$ decays determine the angle $\alpha$ [1]. Using the tree-level UT, we can extract $C_{B_{q}}, \phi_{B_{q}}$ and $C_{\varepsilon_{K}}$ from experimental data $[1,2,3]$.

In Figure 1 we compare the determination of the UT in the presence of NP to the result of the SM UTA. Thanks to the redundancy of the UTA, the accuracy obtained in the presence of NP is comparable to the SM one.

Figure 2 shows the result of the NP analysis for the $K, B_{d}$ and $B_{s}$ sectors. Numerical results for NP parameters are summarized in table 1 . We see full compatibility with the SM, with possible NP amplitudes in the $20-30 \%$ range.

Let us now turn to the analysis of $D-\bar{D}$ mixing. We update the analysis of ref. [4] including the recent $\mathrm{LHCb}$ measurement of $D \rightarrow K \pi$ decays [5]. Since it is a CP-averaged measurement, we interpret it as follows:

$$
y^{\prime} \rightarrow \frac{y_{+}^{\prime}+y_{-}^{\prime}}{2}, \quad\left(x^{\prime}\right)^{2} \rightarrow \frac{\left(x_{+}^{\prime}\right)^{2}+\left(x_{-}^{\prime}\right)^{2}+\left(y_{+}^{\prime}\right)^{2}+\left(y_{-}^{\prime}\right)^{2}}{2}-\left(y^{\prime}\right)^{2} .
$$



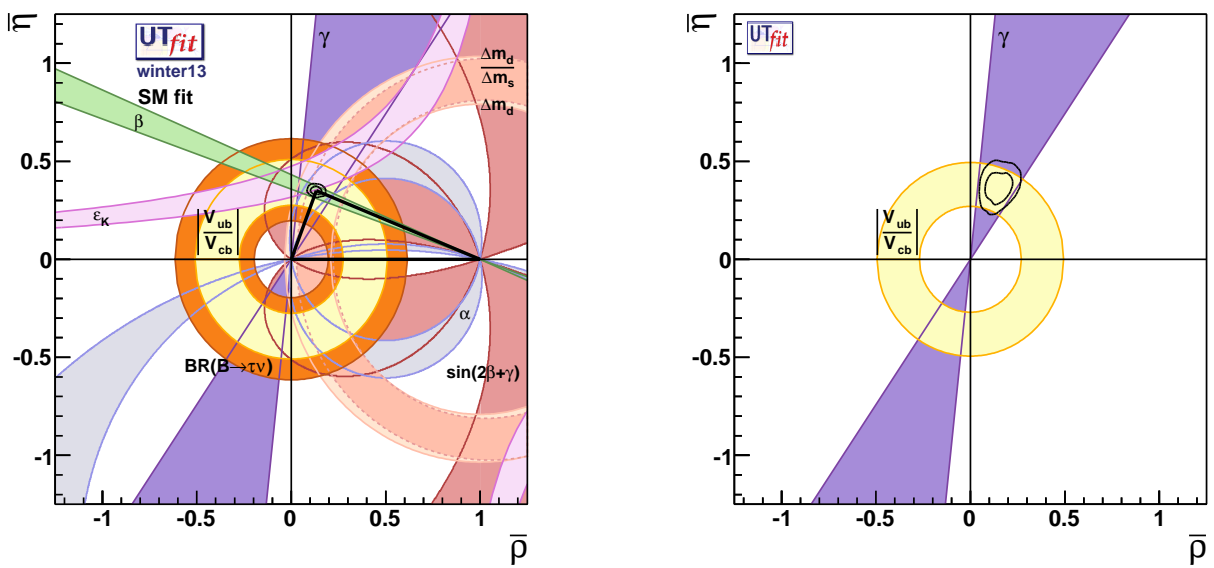

Figure 1: Determination of the UTA within the SM (left) or in the presence of NP (right). The contours correspond to $68 \%$ and $95 \%$ probability regions. The coloured regions correspond to $95 \%$ probability for a single constraint.

The results of the fit are reported in Table 2. The corresponding p.d.f are shown in Figs. 3 and 4.

\section{Constraints on the scale of NP}

Let us now consider the most general form of the $\Delta F=2$ effective weak Hamiltonian. The latter is parameterized by Wilson coefficients of the form

$$
C_{i}(\Lambda)=\frac{F_{i} L_{i}}{\Lambda^{2}}, \quad i=1, \ldots, 5,
$$

where $F_{i}$ is the (generally complex) relevant NP flavor coupling, $L_{i}$ is a (loop) factor which depends on the interactions that generate $C_{i}(\Lambda)$, and $\Lambda$ is the scale of NP, i.e. the typical mass of new particles mediating $\Delta F=2$ transitions. For a generic strongly interacting theory with an unconstrained flavor structure, one expects $F_{i} \sim L_{i} \sim 1$, so that the phenomenologically allowed range for each of the Wilson coefficients can be immediately translated into a lower bound on $\Lambda$. Specific assumptions on the flavor structure of NP correspond to special choices of the $F_{i}$ functions. For example Minimal Flavor Violation (MFV) models $[6,7,8,9]$ correspond to $F_{1}=F_{\mathrm{SM}}$ and $F_{i \neq 1}=0$.

Following Ref. [10], in deriving the lower bounds on the NP scale $\Lambda$, we assume $L_{i}=1$, that corresponds to strongly-interacting and/or tree-level coupled NP. Two other interesting possibilities are given by loop-mediated NP contributions proportional to either $\alpha_{s}^{2}$ or $\alpha_{W}^{2}$. The first case 

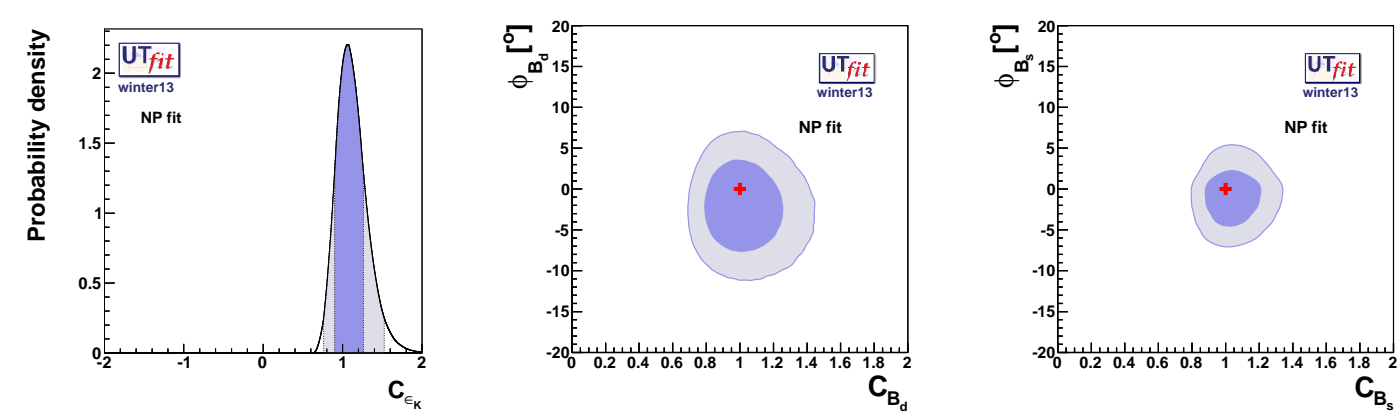

Figure 2: The dark and light colored areas show the $68 \%$ and $95 \%$ probability regions.

\begin{tabular}{|ccc|}
\hline parameter & result @ 68\% prob. & 95\% prob. range \\
\hline$\left|M_{12}\right|[1 / \mathrm{ps}]$ & $(5.0 \pm 2.1) \cdot 10^{-3}$ & {$[0.8,9.1] \cdot 10^{-3}$} \\
$\left|\Gamma_{12}\right|[1 / \mathrm{ps}]$ & $(16 \pm 2) \cdot 10^{-3}$ & {$[12,20] \cdot 10^{-3}$} \\
$\Phi_{12}\left[{ }^{\circ}\right]$ & $(2 \pm 11)$ & {$[-39,35]$} \\
\hline$x$ & $(4.2 \pm 1.8) \cdot 10^{-3}$ & {$[0.4,7.7] \cdot 10^{-3}$} \\
$y$ & $(6.4 \pm 0.8) \cdot 10^{-3}$ & {$[5,8] \cdot 10^{-3}$} \\
$|q / p|-1$ & $(-1.5 \pm 7.7) \cdot 10^{-2}$ & {$[-16,17] \cdot 10^{-2}$} \\
$\phi\left[^{\circ}\right]$ & $(0.3 \pm 2.6)$ & {$[-8.8,3.7]$} \\
\hline$A_{\Gamma}$ & $(-0.1 \pm 0.7) \cdot 10^{-3}$ & {$[-1.6,1.4] \cdot 10^{-3}$} \\
$A_{M}$ & $(3 \pm 15) \cdot 10^{-2}$ & {$[-33,32] \cdot 10^{-2}$} \\
$R_{M}$ & $(2.8 \pm 0.8) \cdot 10^{-5}$ & {$[1.6,5.1] \cdot 10^{-5}$} \\
$R_{D}$ & $(3.47 \pm 0.06) \cdot 10^{-3}$ & {$[3.36,3.59] \cdot 10^{-3}$} \\
$\delta_{K \pi}\left[^{\circ}\right]$ & $(11 \pm 13)$ & {$[-26,34]$} \\
$\delta_{K \pi \pi^{0}}\left[^{\circ}\right]$ & $(33 \pm 21)$ & {$[-10,79]$} \\
\hline
\end{tabular}

Table 2: Results of the fit to $D$ mixing data.

corresponds for example to gluino exchange in the minimal supersymmetric SM. The second case applies to all models with SM-like loop-mediated weak interactions. To obtain the lower bound on $\Lambda$ entailed by loop-mediated contributions, one simply has to multiply the bounds we quote in the following by $\alpha_{s}(\Lambda) \sim 0.1$ or $\alpha_{W} \sim 0.03$.

In agreement with Ref. [10], we find that in the $K^{0}$ sector, due to the non-vanishing chiral limit (chiral enhancement) of their matrix elements, all bounds coming from the contributions of non-standard operators (i.e. from the operators $O_{i}$ with $i \neq 1$ ) are more than one order of magnitude stronger than the bound from the SM $O_{1}$ operator. 

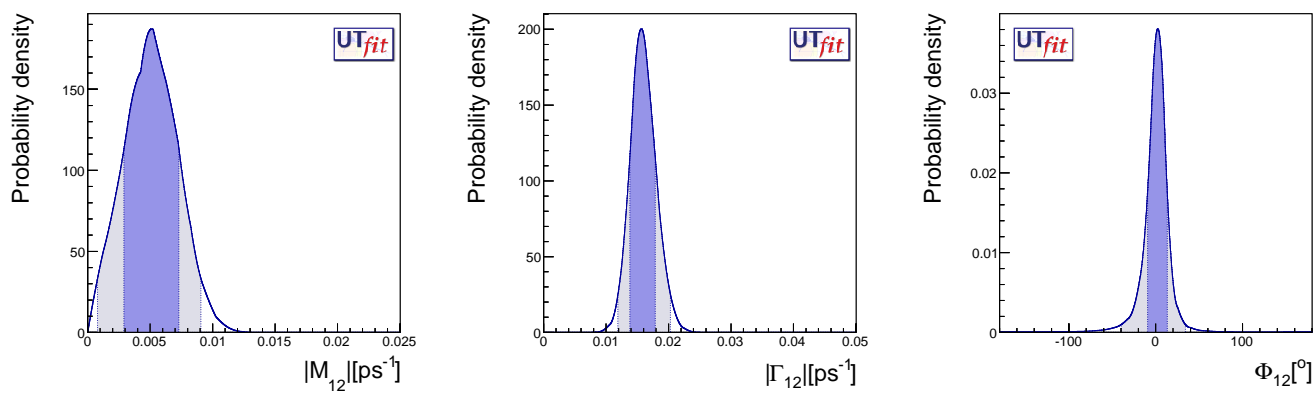

Figure 3: One-dimensional p.d.f. for the parameters $\left|M_{12}\right|,\left|\Gamma_{12}\right|$ and $\Phi_{12}$.
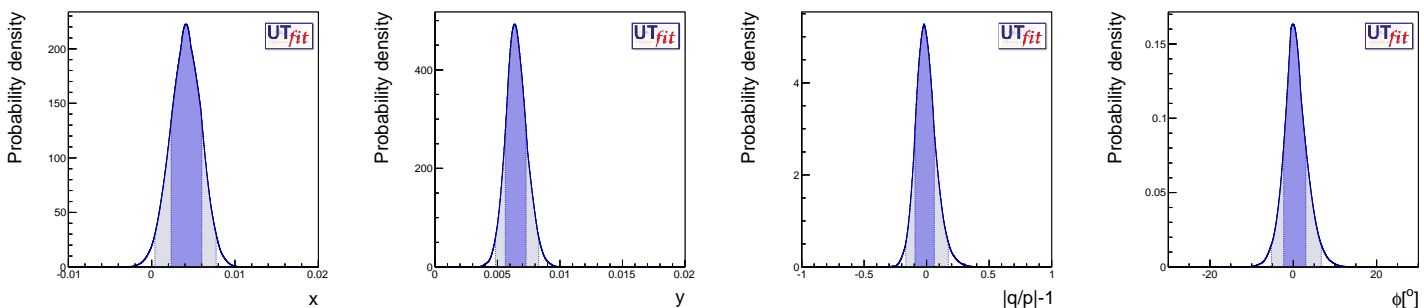

Figure 4: One-dimensional p.d.f. for the parameters $x, y,|q / p|-1$ and $\phi$.

\section{Conclusions}

At present, all observed phenomena in the realm of $\mathrm{CP}$ violation exhibit a remarkable consistency with the SM predictions. Given the loop and GIM suppression of CP violation in the SM, this consistency can be translated into formidable bounds on the scale of NP. It remains as a theoretical challenge to understand the origin of the fermion mass and mixing hierarchy and to provide a suitable protection to NP contributions to CP violation, in such a way that NP can arise at scales relevant for the solution of the hierarchy problem. In this respect, probing flavour and CP violation with increasing accuracy remains the main avenue to physics beyond the SM.

\section{References}

[1] M. Bona et al. [UTfit Collaboration], JHEP 0603 (2006) 080 [arXiv:hep-ph/0509219].

[2] M. Bona et al. [UTfit Collaboration], Phys. Rev. Lett. 97 (2006) 151803 [arXiv:hep-ph/0605213]; JHEP 0803 (2008) 049 [arXiv:0707.0636 [hep-ph]].

[3] M. Bona et al. [UTfit Collaboration], PMC Phys. A3 (2009) 6 [arXiv:0803.0659 [hep-ph]].

[4] A. J. Bevan et al. [UTfit Collaboration], JHEP 1210 (2012) 068 [arXiv:1206.6245 [hep-ph]].

[5] RAaij et al. [LHCb Collaboration], Phys. Rev. Lett. 110 (2013) 101802 [arXiv:1211.1230 [hep-ex]].

[6] M. Misiak, S. Pokorski and J. Rosiek, Adv. Ser. Direct. High Energy Phys. 15 (1998) 795 [hep-ph/9703442].

[7] M. Ciuchini, G. Degrassi, P. Gambino and G. F. Giudice, Nucl. Phys. B 534 (1998) 3 [hep-ph/9806308]. 


\begin{tabular}{|ccc|}
\hline \hline & $\begin{array}{c}95 \% \text { allowed range } \\
\left(\mathrm{GeV}^{-2}\right)\end{array}$ & $\begin{array}{c}\text { Lower limit on } \Lambda \\
(\mathrm{TeV})\end{array}$ \\
\hline $\operatorname{Im} C_{1}^{K}$ & {$[-2.1,3.4] \cdot 10^{-15}$} & $1.7 \cdot 10^{4}$ \\
$\operatorname{Im} C_{2}^{K}$ & {$[-2.1,1.4] \cdot 10^{-17}$} & $22 \cdot 10^{4}$ \\
$\operatorname{Im} C_{3}^{K}$ & {$[-5.1,7.8] \cdot 10^{-17}$} & $11 \cdot 10^{4}$ \\
$\operatorname{Im} C_{4}^{K}$ & {$[-3.0,4.7] \cdot 10^{-18}$} & $46 \cdot 10^{4}$ \\
$\operatorname{Im} C_{5}^{K}$ & {$[-0.9,1.4] \cdot 10^{-17}$} & $27 \cdot 10^{4}$ \\
\hline $\operatorname{Abs} C_{1}^{B_{d}}$ & $<4.7 \cdot 10^{-12}$ & $4.6 \cdot 10^{2}$ \\
$\operatorname{Abs} C_{2}^{B_{d}}$ & $<3.0 \cdot 10^{-13}$ & $1.8 \cdot 10^{3}$ \\
$\operatorname{Abs} C_{3}^{B_{d}}$ & $<1.1 \cdot 10^{-12}$ & $9.5 \cdot 10^{2}$ \\
$\operatorname{Abs} C_{4}^{B_{d}}$ & $<9.5 \cdot 10^{-14}$ & $3.2 \cdot 10^{3}$ \\
$\operatorname{Abs} C_{5}^{B_{d}}$ & $<2.7 \cdot 10^{-13}$ & $1.9 \cdot 10^{3}$ \\
\hline $\operatorname{Abs} C_{1}^{B_{s}}$ & $<5.6 \cdot 10^{-11}$ & $1.3 \cdot 10^{2}$ \\
$\operatorname{Abs} C_{2}^{B_{s}}$ & $<4.9 \cdot 10^{-12}$ & $4.5 \cdot 10^{2}$ \\
$\operatorname{Abs} C_{3}^{B_{s}}$ & $<1.8 \cdot 10^{-11}$ & $2.3 \cdot 10^{2}$ \\
$\operatorname{Abs} C_{4}^{B_{s}}$ & $<1.6 \cdot 10^{-12}$ & $7.9 \cdot 10^{2}$ \\
$\operatorname{Abs} C_{5}^{B_{s}}$ & $<4.5 \cdot 10^{-12}$ & $4.7 \cdot 10^{2}$ \\
\hline $\operatorname{Im} C_{1}^{D}$ & {$[-2.1,1.7] \cdot 10^{-13}$} & $2.2 \cdot 10^{3}$ \\
$\operatorname{Im} C_{2}^{D}$ & {$[-1.9,2.3] \cdot 10^{-14}$} & $6.6 \cdot 10^{3}$ \\
$\operatorname{Im} C_{3}^{D}$ & {$[-7.1,5.8] \cdot 10^{-13}$} & $1.2 \cdot 10^{3}$ \\
$\operatorname{Im} C_{4}^{D}$ & {$[-6.6,5.3] \cdot 10^{-15}$} & $1.2 \cdot 10^{4}$ \\
$\operatorname{Im} C_{5}^{D}$ & {$[-8.8,7.1] \cdot 10^{-14}$} & $3.4 \cdot 10^{3}$ \\
\hline
\end{tabular}

Table 3: $95 \%$ probability range for the coefficients and the corresponding lower bounds on the NP scale, $\Lambda$, for a generic strongly interacting NP with generic flavor structure $\left(L_{i}=F_{i}=1\right)$.

[8] A. J. Buras, P. Gambino, M. Gorbahn, S. Jager and L. Silvestrini, Phys. Lett. B 500 (2001) 161 [hep-ph/0007085].

[9] G. D’Ambrosio, G. F. Giudice, G. Isidori and A. Strumia, Nucl. Phys. B 645 (2002) 155 [hep-ph/0207036].

[10] M. Bona et al. [UTfit Collaboration], JHEP 0803 (2008) 049 [arXiv:0707.0636 [hep-ph]]. 